\section{A219 REGULATION OF VLA-4 IN LUPUS MONOCYTES OBJECTIVES}

Homaira Rahami Pediatrics/Rheumatology, Children's Hospital of Philadelphia, Pennsylvania, USA

10.1136/ard.2011.151209.4

Objectives The development of atherosclerosis and subsequent increased risk of cardiovascular disease (CVD) is known to be associated with inflammation. Studies have shown an increased risk of CVD in systemic lupus erythematosus (SLE). Inflammation seen in atherosclerosis involves various immune mediators, including interactions between adhesion molecules on endothelial cells and on monocytes. Very late antigen-4 (VLA-4) is an adhesion molecule in the integrin family found on monocytes and has been implicated in monocyte recruitment during development of atherosclerosis. A recent study noted an increased expression of VLA-4 in SLE monocytes, but the regulation is poorly understood. We aimed to further define variables governing the expression of VLA-4 on monocytes in patients with SLE compared to healthy controls.

Methods CD14+ human monocytes were obtained from whole blood of healthy adult patients and SLE patients. Flow cytometry was used to measure expression of VLA-4 at rest and upon exposure to various agents, including interferon $\gamma$, interferon $\alpha$, interleukin- 4 and vitamin D. VLA-4 mRNA expression in SLE and healthy monocytes was measured using qRT-PCR. Healthy monocytes were incubated in control and SLE sera, and VLA-4 was expression evaluated using flow cytometry and immunofluorescence (IF).

Results VLA-4 (CD49d) expression was significantly higher on control monocytes compared to SLE monocytes as measured by mean fluorescence intensity $(p<0.05)$. However, levels of VLA-4 mRNA were significantly lower in control monocytes compared to SLE monocytes $(p<0.05)$. Exposure of healthy monocytes to healthy human sera compared to SLE sera did not result in differences in cell surface expression of VLA-4; however, VLA-4 was redistributed to the interior of the cell. IF staining of VLA-4 of healthy monocytes incubated in SLE sera demonstrated VLA- 4 in a vesicular compartment whereas healthy monocytes incubated in healthy sera or RPMI media alone exhibited VLA-4 staining on the surface or diffusely in the cytoplasm.

Conclusion There was a significant downregulation of cell surface expression of VLA- 4 in SLE monocytes as compared to the healthy population although mRNA levels were increased. We demonstrated that exposure to SLE sera causes increased internal IF staining of VLA-4, suggesting that decreased surface expression, in spite of increased RNA levels, in SLE is due to altered distribution. VLA-4 has increased expression in atherosclerosis, and understanding its upregulation in SLE patients could have implications for the prevention and treatment of advanced atherosclerosis in SLE. In addition, VLA-4 is capable of intracellular signalling. If there is an altered distribution of VLA-4 in SLE monocytes, this might affect the inflammatory pathway in SLE. These data provide a context for considering novel therapeutics to treat atherosclerosis in SLE. 


\section{Corrections}

Homaira Rahami. Regulation of VLA-4 in lupus monocytes objectives. Ann Rheum Dis 2011;70:A94. The name of the author Homaira Rahimi was incorrectly published as Homaira Rahami. Also, the following authors and their affiliations were missing from the author list. Kathleen Sullivan, The Children's Hospital of Philadelphia, Philadelphia, USA. Michelle Petri, Johns Hopkins University, Baltimore, Maryland, USA.

Ann Rheum Dis 2011;70:2060. doi:10.1136/ard.2011.151209.4corr1 